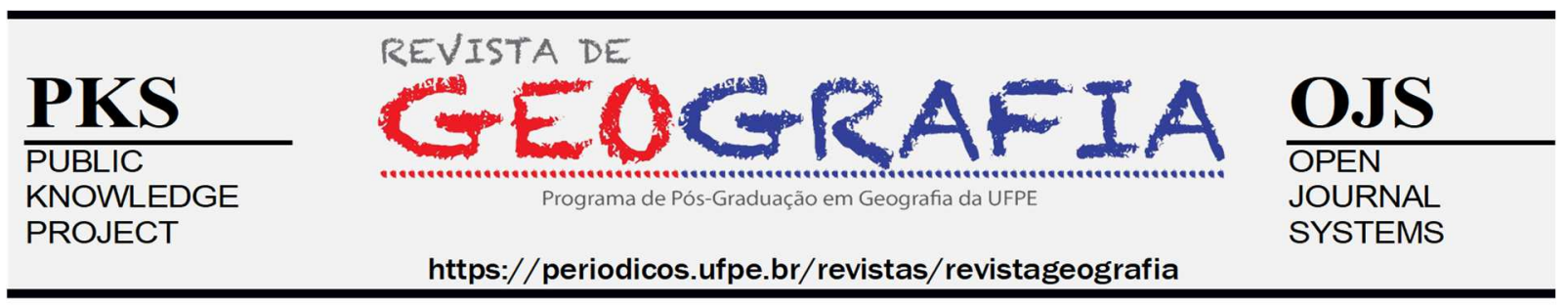

\title{
USO DE TELHADOS VERDES PARA CONTROLE DE ÁGUAS PLUVIAIS URBANAS EM TERESINA - PIAUÍ
}

\author{
Mauro César de Brito Sousa ${ }^{1}$, Renato Alves Pedrosa ${ }^{2}$, Bruna de Freitas Iwata ${ }^{3}$, Sammya \\ Vanessa Vieira Chaves ${ }^{4}$
}

\begin{abstract}
${ }^{1}$ Instituto Federal do Piauí, Engenheiro Civil, Doutor em Engenharia Civil (Recursos Hídricos), Professor do Mestrado em Análise e Planejamento Espacial (IFPI), E-mail: mauro.sousa@ifpi.edu.br, ORCID: http://orcid.org/0000-0002-0650-6577

${ }^{2}$ Instituto Federal do Maranhão, Engenheiro Civil, Professor do IFMA, Mestrando em Análise e Planejamento Espacial (IFPI), E-mail: renatopedrosa @ hotmail.com, ORCID: http://orcid.org/0000-0002-2366-9239.

${ }^{3}$ Instituto Federal do Piauí, Gestora Ambiental, Doutora em Ciências do Solo, Professora do Mestrado em Análise e Planejamento Espacial (IFPI), E-mail: iwata@ifpi.edu.br, ORCID: http://orcid.org/0000-0002-6465-9731

${ }^{4}$ Instituto Federal do Piauí, Geógrafa, Doutora em Geografia, Professora do Mestrado em Análise e Planejamento Espacial (IFPI), E-mail: sammyachaves@ @ifpi.edu.br, ORCID: https://orcid.org/0000-0002-9763-3079
\end{abstract}

Artigo recebido em 08/06/2020 e aceito em 06/05/2021

\section{RESUMO}

Ao longo dos últimos anos, o meio científico tem exposto suas críticas quanto à flexibilidade limitada das soluções convencionais de drenagem no combate às inundações urbanas. Atualmente, novas tecnologias têm surgido com intuito de garantir soluções sustentáveis ao gerenciamento urbano de águas pluviais, sendo chamadas de Desenvolvimento Urbano de Baixo Impacto (Low Impact DevelopmentLID). Este estudo avaliou o uso desses dispositivos na bacia hidrográfica PD14 localizada na cidade de Teresina, Piauí, observando os efeitos de sua implementação para mitigação de escoamentos superficiais. O software PCSWMM foi utilizado para modelagem de LIDs do tipo telhado verde na área de estudo, considerando dois cenários diferentes de abrangência das LIDs modeladas. A partir da análise dos cenários modelados, observou-se a redução do volume total de água escoada variando entre 4,7 \% e $12,13 \%$, além da redução do pico de escoamento e atraso no início do deflúvio. Os resultados demonstraram a utilidade prática do uso de telhados verdes como medida eficaz de controle e mitigação do escoamento superficial em bacias urbanas.

Palavras-chave: drenagem urbana; desenvolvimento de baixo impacto; telhados verdes. 


\title{
THE USE OF GREEN ROOFS TO CONTROL URBAN STORMWATER IN TERESINA - PIAUÍ
}

\begin{abstract}
Over the last years, researchers have posed their criticism regarding the limited flexibility of conventional drainage solutions to flooding. Nowadays, new technologies have emerged that take into account the sustainable solutions of urban stormwater management and they are called Low Impact Development (LID). This study evaluates the use of these devices in watersheds located in the city of Teresina, Piauí, indicating the efficiency of the method to mitigate surface runoff. PCSWMM software was used to model green roof LIDs in the study area considering two different scenarios of the modeled LIDs. From the analysis of the modeled scenarios, there was a decline in the total volume of runoff water varying between $4.7 \%$ and $12.13 \%$, in addition to the declinein the runoff peak and delay in the beginning of the defluvium. The results demonstrated the practical utility of using green roofs as an effective measure to control and mitigate runoff in urban basins.
\end{abstract}

Keywords: urban drainage; low impact development; green roofs.

\section{EL USO DE CUBIERTAS VERDES PARA CONTROLAR LAS AGUAS PLUVIALES URBANAS EN TERESINA (PIAUÍ)}

\begin{abstract}
RESUMEN
En los últimos años, la comunidad científica ha criticado la escasa flexibilidad de las soluciones convencionales de drenaje para combatir las inundaciones urbanas. Actualmente, están surgiendo nuevas tecnologías que ofrecen soluciones sostenibles para la gestión de las aguas pluviales urbanas, a las que se denomina «de Desarrollo Urbano de Bajo Impacto (LID, del inglés "Low Impact Development")». Este estudio ha evaluado el uso de estos dispositivos en la cuenca hidrográfica PD14, ubicada en la ciudad de Teresina, en Piauí, observando los efectos de su implementación para la mitigación de las escorrentías superficiales. El «software» PCSWMM se empleó para modelar desarrollos de bajo impacto (LID) de cubiertas verdes en la zona de estudio, considerando dos escenarios diferentes de alcance de los desarrollos modelados. A partir del análisis de los escenarios modelados, se ha observado una reducción en el volumen total del agua escurrida, con una variación de entre el 4,7 \% y el $12,13 \%$, además de una reducción del pico de escorrentía y un retraso en el inicio del escurrimiento. Los resultados demuestran la utilidad práctica del uso de cubiertas verdes como medida eficaz para controlar y mitigar la escorrentía en las cuencas urbanas.
\end{abstract}

Palabras clave: drenaje urbano; desarrollo de bajo impacto; cubiertas verdes. 


\section{INTRODUÇÃO}

O crescente agravamento da falta de controle do uso e ocupação do solo e constante impermeabilização de áreas de bacias urbanas trazem consequências hidrológicas indesejáveis, como o aumento do escoamento superficial que sobrecarrega o sistema de drenagem, ocasionando inúmeros desafios para gestores, pesquisadores e comunidade afetada (PALLA et al., 2018).

Em face dos efeitos colaterais das soluções até então adotadas, as técnicas de drenagem urbana têm gradualmente avançado para práticas de manejo de águas urbanas e inserido em seu arcabouço de soluções conceitos que estruturem o espaço em conformidade com o ciclo hidrológico natural da bacia hidrográfica (MIGUEZ et al., 2016).

Segundo Miguez et al. (2018) e Kourtis et al. (2018), os conceitos inovadores mais adotados para a readequação dos sistemas de drenagem têm por objetivo retardar o escoamento, de forma a propiciar o aumento dos tempos de concentração e a consequente redução nas vazões máximas.

Surgem, portanto, tecnologias que consideram outros aspectos do gerenciamento de águas pluviais, como: a qualidade do escoamento superficial; os aspectos paisagísticos; o valor recreativo; e a proteção ecológica da bacia. Essas soluções de drenagem têm sido amplamente aplicadas em diferentes partes do mundo e são denominadas de Soluções de Desenvolvimento de Baixo Impacto ou LID (Low Impact Development) (KOURTIS et al., 2018).

$\mathrm{Na}$ China, por exemplo, o termo Cidade Esponja reflete um esforço implementado desde 2014 para alterar o paradigma de projetos de drenagem no país. Deste modo, células de bioretenção, telhados verdes, pavimentos porosos, lagoas de detenção e áreas úmidas (wetlands) são práticas usualmente concebidas e representativas das Soluções de Desenvolvimento de Baixo Impacto que obrigam os espaços urbanos a funcionarem como esponjas que retêm e liberam paulatinamente a água das chuvas (MAO et. al, 2016).

Conforme entendimento de CIRIA (2015), o uso de LIDs pode melhorar a resiliência das cidades a cheias e alagamentos, configurando-se em uma resposta consistente para a variabilidade climática que aflige as soluções tradicionais de drenagem. 
Este estudo avalia o uso de soluções de Desenvolvimento Urbano de Baixo Impacto (LIDs), do tipo telhado verde, em bacias hidrográficas na cidade de Teresina, Piauí, observando os efeitos de sua implementação na mitigação de deflúvios urbanos.

\section{MATERIAIS E MÉTODO}

Para realização do estudo, utilizou-se o processo de modelagem apontado por Kourtins et. al (2018), que considera a escolha do modelo adequado para a resposta hidrológica do sistema, as características da LID a ser modelada e a confecção de cenários com e sem a implementação de telhados verdes para comparação.

Assim, primeiramente foi definida as características fisiográficas da bacia de estudo e meteorológicas locais, para delimitação das condições hidrológicas do cenário inicial. Posteriormente, fez-se a caracterização da LID a ser modelada, conforme parâmetros de entrada específicos do modelo chuva-vazão escolhido. Finalmente, simulou-se o runoff na bacia, considerando a contraposição do cenário inicial e cenários com LIDs modeladas.

\section{Área de estudo}

A bacia selecionada para o estudo foi a sub-bacia PD14, localizada em um segmento retilíneo do rio Poti, na parte central da cidade de Teresina, estado do Piauí, sendo uma região urbanizada, com gradiente topográfico suave e propícia a alagamentos devido à ausência de sistemas de drenagem adequados às necessidades de escoamento das vazões geradas na subbacia (TERESINA, 2010). A Figura 1 apresenta a Bacia PD14 modelada neste trabalho. 
Figura 1 - Sub-Bacia PD14, Teresina - Piauí

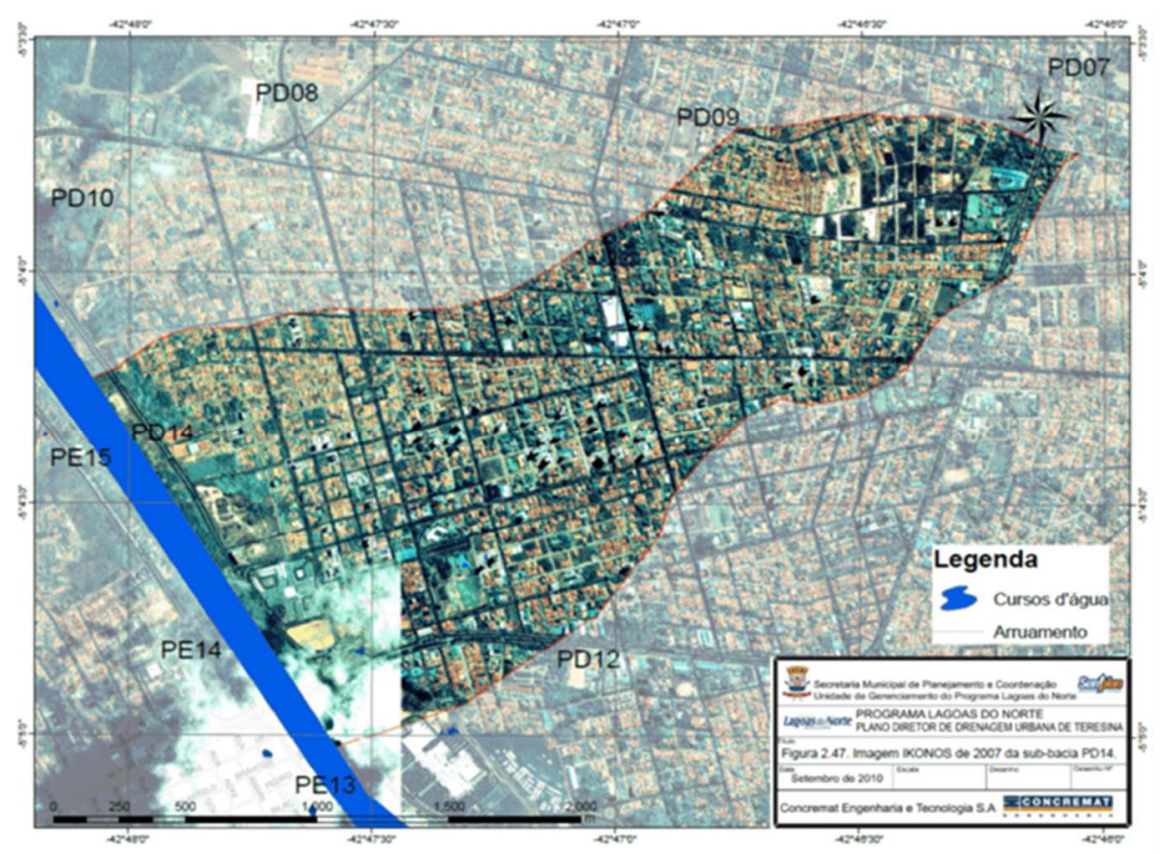

Fonte: Teresina (2010)

A bacia teve suas características fisiográficas levantadas pelo Plano Diretor de Drenagem Urbana de Teresina (PDDUr) (TERESINA, 2010), indicando, dentre outros dados pertinentes ao modelo: a área de 4,25 km²; o tempo de concentração da bacia calculado em 14,73 min; a área impermeável estimada em torno de 62,45 \%; e o CN médio de 88,97.

\section{Dados de precipitação}

A equação genérica para as curvas de Intensidade-Duração-Frequência (IDF) de Teresina (Equação 1) também foi determinada pelo PDDUr e foi utilizada para definir o hietograma de entrada do modelo chuva-vazão para a área modelada.

$$
i=\frac{1194,273 \times T^{0,1738}}{(t+10)^{0,7457}}
$$

Sendo i a intensidade da chuva em $\mathrm{mm} / \mathrm{h}$, $\mathbf{T}$ o período de retorno em anos e $\mathbf{t}$ o tempo de concentração da bacia em min. O intervalo de tempo considerado para a discretização da chuva de projeto foi determinado em função do menor tempo de concentração encontrado para as sub-bacias existentes dentro da área modelada PD14. Assim, considerou-se para o hietograma resultante (Figura 2), o tempo de concentração de 2 (dois) min, o tempo de 
recorrência de 5 (cinco) anos (tempo base para projetos de drenagem urbana) e o tempo de observação do evento chuvoso de 1 hora.

Figura 2 - Hietograma para Sub - Bacia PD 14

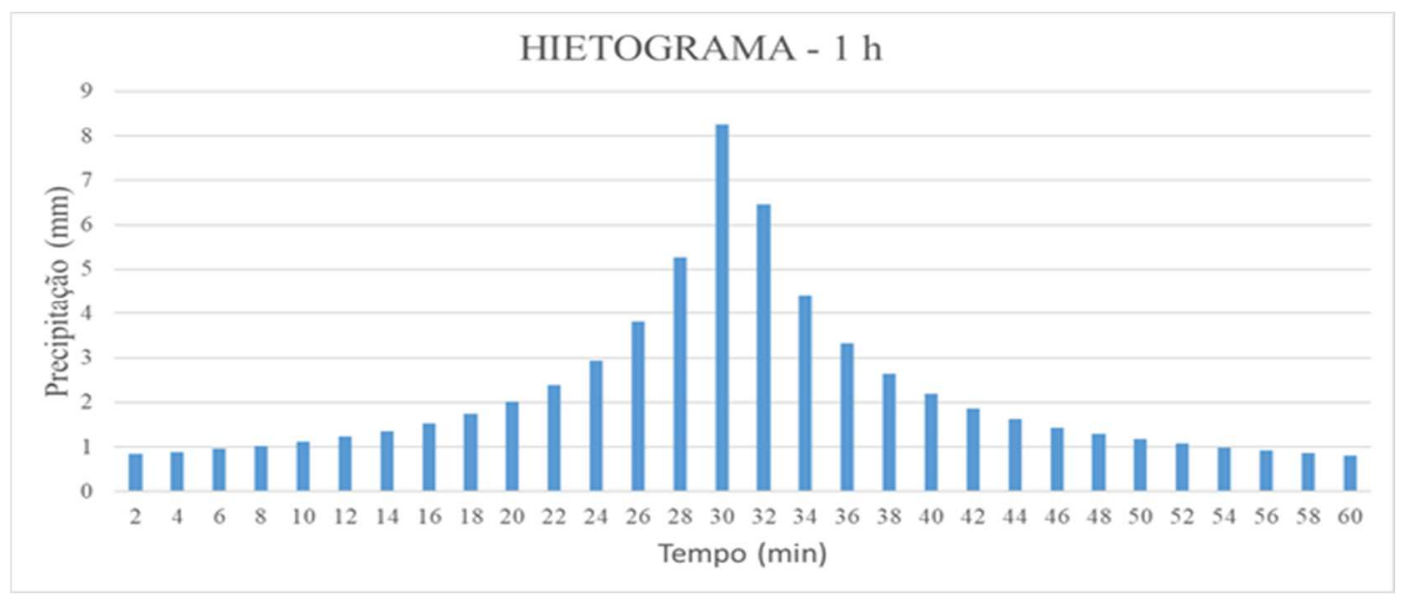

Fonte: Autores, 2020.

Modelagem dos telhados verdes

Considerando a classificação de Martins (2017), os telhados verdes extensivos foram selecionados como opção de investigação, por apresentarem facilidades de instalação, serem mais leves, baratos e exigirem pequena ou nenhuma manutenção. Na Tabela 1 encontram-se os dados dos telhados modelados.

Tabela 1 - Dados de entrada para modelagem do telhado verde

Unidad

\begin{tabular}{cccc} 
Camada & Parâmetro & e & Valor \\
\hline \multirow{3}{*}{ Superfície } & Armazenamento & $\mathrm{mm}$ & 0,00 \\
& Volume de vegetação & $\%$ & 0,90 \\
& Rugosidade (n) & - & 0,10 \\
& Declividade & $\%$ & 20,00 \\
\hline \multirow{4}{*}{ Solo } & Espessura & $\mathrm{mm}$ & 150,00 \\
& Porosidade & $\%$ & 0,437 \\
& Capacidade de campo & $\mathrm{mm}$ & 0,105 \\
& Ponto de murcha & $\%$ & 0,047 \\
& Condutividade & $\mathrm{mm} / \mathrm{hr}$ & 3,00 \\
& Condutividade & $\%$ & 10,00 \\
& Sucção capilar & $\mathrm{mm}$ & 2,40 \\
\hline \multirow{4}{*}{ Dreno } & Coeficiente de & & \\
(manta de & drenagem & - & 3,00 \\
drenagem) & Fração de vazios & - & 0,50 \\
& Rugosidade n & $\mathrm{mm}$ & 0,10 \\
\hline & Fonte: Martins (2017) &
\end{tabular}


Para a modelagem da LID escolhida, limitou-se à criação de dois cenários com área presumível de telhados verdes. O primeiro cenário foi definido com uma área de cobertura por telhados verdes de $75 \%$ e o segundo cenário com $10 \%$, e então avaliou-se a interferência do percentual de LID no pico e volume de escoamento da bacia analisada.

Nesse caso, a área total de telhados na bacia foi estimada em 138,49 ha, perfazendo $32,6 \%$ da área total da bacia PD14. Desses 138,49 ha, estima-se uma área de 103,87 ha para o cenário com $75 \%$ de telhados verdes, e uma área de 13,85 ha para o cenário com $10 \%$ de telhados verdes, conforme resumo no Quadro 1.

Quadro 1 - Áreas de telhados na bacia PD14

\begin{tabular}{|c|c|c|c|}
\hline Área da Bacia PD14 & $\begin{array}{c}\text { Área total de } \\
\text { telhados }\end{array}$ & $\begin{array}{c}\text { Cenário } 1-75 \% \\
\text { de Telhados } \\
\text { Verdes }\end{array}$ & $\begin{array}{c}\text { Cenário } 2-10 \% \\
\text { de Telhados } \\
\text { Verdes }\end{array}$ \\
\hline 425 ha & 138,49 ha & 103,87 há & 13,85 ha \\
\hline
\end{tabular}

Fonte: Autores, 2020.

\section{Modelo chuva-vazão}

Neste trabalho adotou-se o modelo PCSWMM, que apresenta uma combinação adequada entre SIG e US EPA SWMM 5 para modelagem da resposta hidrológica da bacia e tecnologias de controle de águas pluviais, como os telhados verdes.

Para estimar as perdas de infiltração e o escoamento superficial no modelo foram usados o método do Número da Curva (CN) do Serviço de Conservação do Solo (SCS) e a equação de Manning. Além disso, padronizou-se a Teoria da Onda Dinâmica para o cálculo do roteamento de fluxo.

\section{RESULTADOS E DISCUSSÃO}

Resultados da simulação do escoamento na fase diagnóstico (sem LID)

Considerando a modelagem do cenário de controle sem a implementação de telhados verdes instalados na bacia PD14, observa-se que o início do escoamento superficial acontece no primeiro minuto de simulação, atingindo o seu pico aos 00:32:00 minutos e valor calculado em 147.860,60 L/s. O volume de água escoado durante o evento chuvoso modelado foi de 
$228.600 \mathrm{~m}^{3}$. O Quadro 2 apresenta o resumo dos resultados obtidos na simulação e comportamento da vazão no tempo de simulação expresso na figura 3.

Quadro 2 - Simulação do escoamento superficial na fase diagnóstico

\begin{tabular}{|c|c|c|c|c|}
\hline Bacia Modelada & $\begin{array}{c}\text { Cenário } \\
\text { modelado }\end{array}$ & $\begin{array}{c}\text { Pico de } \\
\text { escoamento } \\
(\mathrm{L} / \mathrm{s})\end{array}$ & $\begin{array}{c}\text { Tempo de } \\
\text { simulação até o } \\
\text { pico }(\mathrm{min})\end{array}$ & $\begin{array}{c}\text { Volume } \\
\text { escoado } \\
\left(\mathrm{m}^{3}\right)\end{array}$ \\
\hline PD14 & $\begin{array}{c}\text { Sem telhados } \\
\text { verdes }\end{array}$ & $147.869,60$ & $00: 32: 00$ & 228.600 \\
\hline
\end{tabular}

Fonte: Autores, 2020.

Figura 3 - Escoamento superficial x Tempo simulado

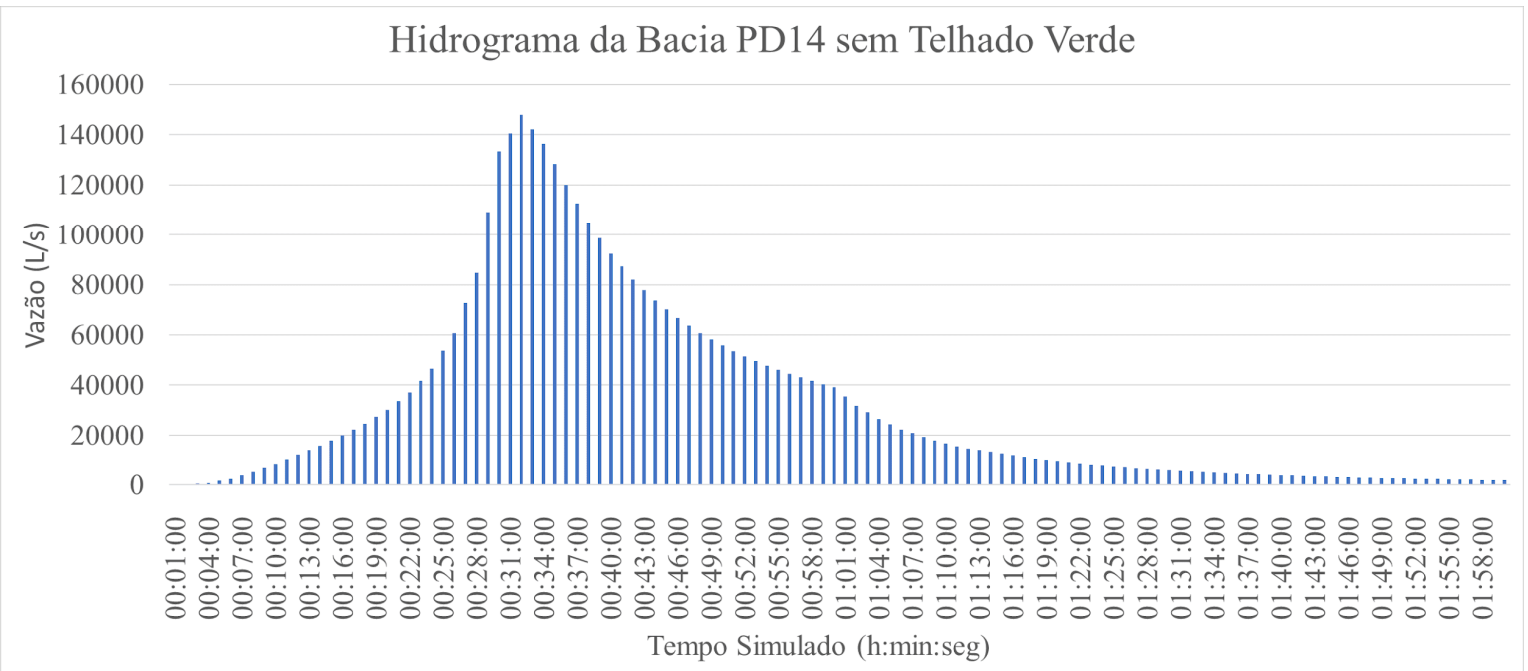

Fonte: Autores, 2020.

Resultados da simulação para cenário com $75 \%$ de área de telhados configurados como telhado verde

O cenário simulado apresenta a perspectiva de que $75 \%$ das áreas de telhados identificadas na sub-bacia PD14 sejam de telhados verdes. Para essa hipótese, o pico de escoamento superficial (runoff) encontrado foi da ordem de 90.398,02 L/s, ocorrendo aos 00:40:00 minutos de simulação e o volume total de água escoada na bacia foi de $200.862 \mathrm{~m}^{3}$, conforme o resumo dos resultados no Quadro 3. 
Quadro 3 - Simulação do escoamento superficial - $75 \%$ dos telhados com LID

\begin{tabular}{|c|c|c|c|c|}
\hline Bacia Modelada & $\begin{array}{c}\text { Cenário dos } \\
\text { telhados } \\
\text { modelado }\end{array}$ & $\begin{array}{c}\text { Pico de } \\
\text { escoamento } \\
(\mathrm{L} / \mathrm{s})\end{array}$ & $\begin{array}{c}\text { Tempo de } \\
\text { simulação até o } \\
\text { pico (min) }\end{array}$ & $\begin{array}{c}\text { Volume } \\
\text { escoado } \\
\left(\mathrm{m}^{3}\right)\end{array}$ \\
\hline PD14 & $\begin{array}{c}75 \% \text { de } \\
\text { telhados } \\
\text { verdes }\end{array}$ & $90.398,02$ & $00: 40: 00$ & 200.862 \\
\hline
\end{tabular}

Fonte: Autores, 2020.

Nesse ponto, percebeu-se uma sensível mudança nos valores de deflúvio identificados na bacia. O pico do escoamento superficial foi reduzido em $38,86 \%$ se comparado ao escoamento gerado na bacia sem a presença de telhados verdes. O volume total de água escoado foi reduzido em $12,13 \%$.

É possível identificar nos dados elencados na Figura 4 que o escoamento na bacia começa com atraso de 16 minutos se comparado ao cenário anteriormente modelado. Isso se deve ao fato de que os telhados verdes absorvem e direcionam o escoamento para as áreas permeáveis da bacia e somente após a sua capacidade ser suplantada, inicia-se o processo de escoamento superficial.

Figura 4 - Escoamento superficial x Tempo simulado

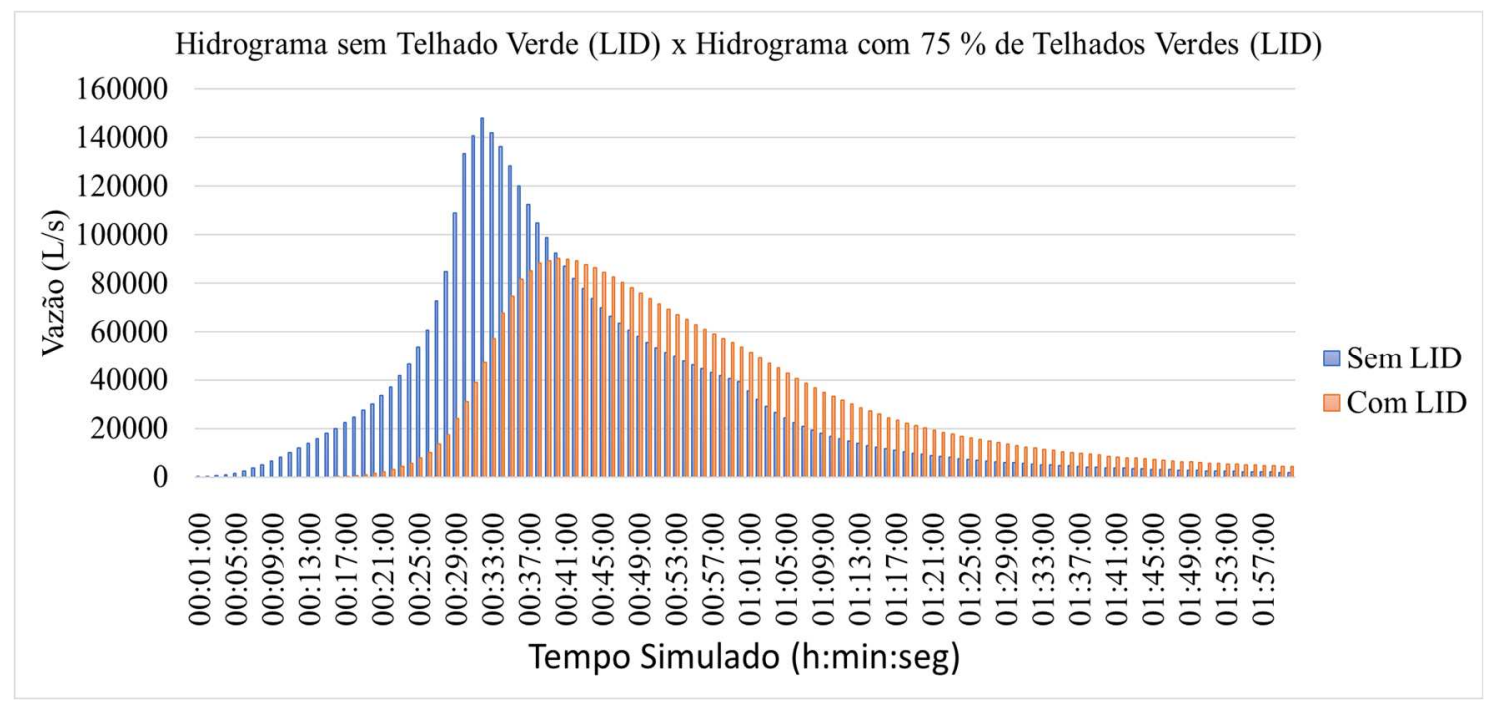

Fonte: Autores, 2020. 
Essa peculiaridade aponta para a capacidade dos telhados verdes modelados atuarem na retenção do escoamento, incrementando um fator de resiliência para a bacia PD14. Além disso, ao demorar a atingir o pico de escoamento, que ocorre somente aos 40 min, evita-se que grandes quantidades de deflúvio cheguem rapidamente ao sistema de drenagem, gerando pontos de alagamento.

A Figura 4 também apresenta a comparação entre os hidrogramas dos cenários que contam com a presença e ausência de telhados verdes na modelagem, onde pode ser constatado a redução do volume escoado, o atraso no início do escoamento e o atraso no pico de escoamento.

Resultados da simulação para cenário com $10 \%$ de área de telhados configurados como telhado verde

No último cenário modelado, apenas $10 \%$ da área considerada de telhados para a bacia PD14 foi transformada em telhados verdes.

Apesar disso, obteve-se uma sensível redução do pico do escoamento, com valores estabelecidos em 102.978,93 L/s. O resultado evidencia a redução do pico de deflúvio em termos percentuais de $30 \%$, acontecendo aos 40 min do evento chuvoso. Também é possível observar que a resposta hidrológica na bacia ocorre com atraso de 15 min se comparado ao cenário sem telhado verde.

O volume de deflúvio escoado, segundo os dados simulados, foi de $217.850 \mathrm{~m}^{3}$, demonstrando uma redução de 4,7\% se comparado ao cenário sem LID. O Quadro 4 apresenta o resumo dos dados gerados nessa simulação. A Figura 5 apresenta a comparação entre todos os cenários modelados.

Quadro 4 - Simulação do escoamento superficial - $10 \%$ dos telhados com LID

\begin{tabular}{|c|c|c|c|c|}
\hline Bacia Modelada & Cenário modelado & $\begin{array}{c}\text { Pico de } \\
\text { escoamento } \\
(\mathrm{L} / \mathrm{s})\end{array}$ & $\begin{array}{c}\text { Tempo de } \\
\text { simulação até o } \\
\text { pico }(\mathrm{min})\end{array}$ & $\begin{array}{c}\text { Volume } \\
\text { escoado } \\
\left(\mathrm{m}^{3}\right)\end{array}$ \\
\hline PD14 & $\begin{array}{c}10 \% \text { de telhados } \\
\text { verdes }\end{array}$ & $102.978,93$ & $00: 40: 00$ & 217.850 \\
\hline
\end{tabular}

Fonte: Autores, 2020. 
Figura 5 - Escoamento superficial considerando os cenários simulados

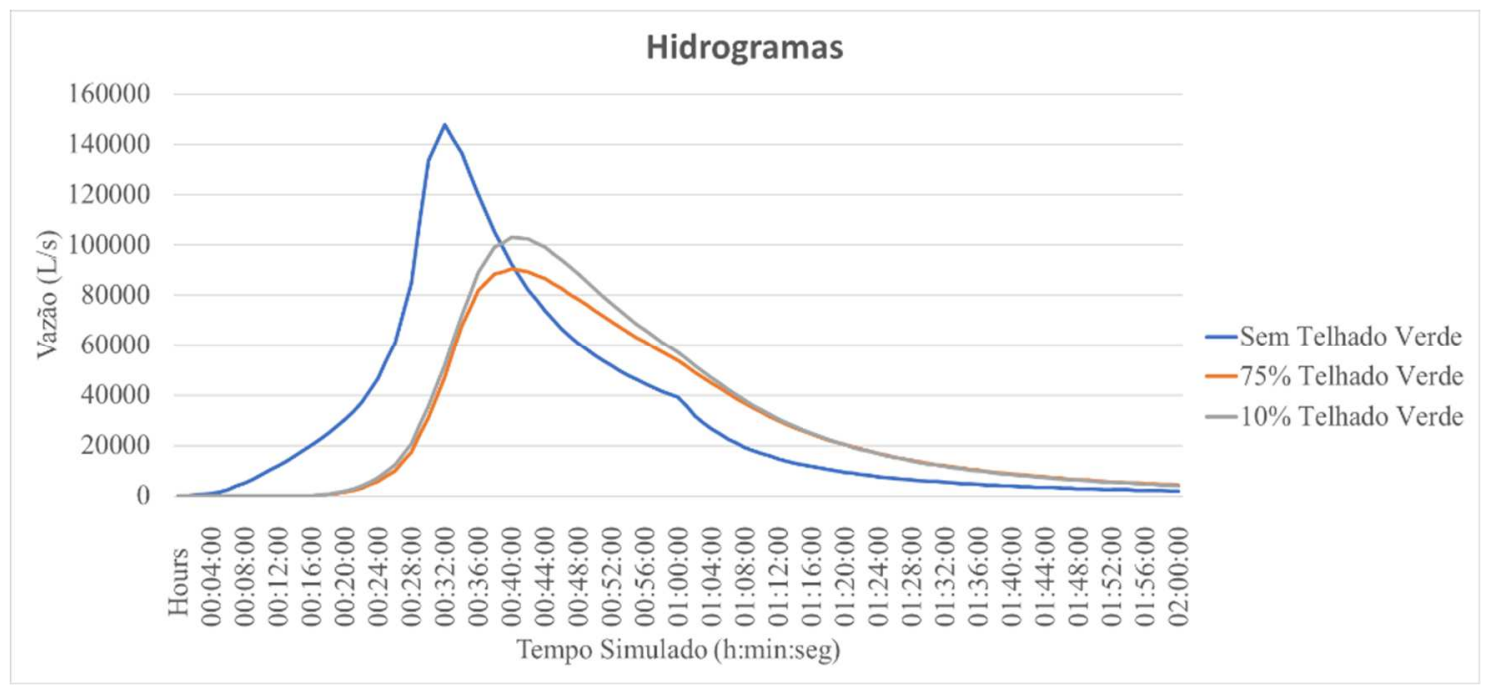

Fonte: Autores, 2020.

Implicações

Após disposição dos dados de redução do volume total de escoamento, retardo do tempo de pico e a mitigação da vazão de escoamento nos diferentes cenários simulados, verificou-se que existe certa dificuldade de se comparar com resultados de estudos anteriores, devido às diferentes condições, propriedades e premissas de modelagem apresentadas.

Essa perspectiva está de acordo com o estudo apresentado por Mora-Melià et al. (2018), que ao utilizar o SWMM para modelar a incorporação de telhados verdes com diferentes porcentagens de área de cobertura em microbacias em Curicó (Chile), indicou que critérios de modelagem adotados influenciam significativamente no desempenho dos telhados verdes e podem dificultar o exercício de comparação com estudos prévios.

Conforme Mora-Melià et al. (2018), estudos anteriores tendem a analisar cenários onde os telhados verdes ocupam 100\% da superfície disponível das sub-bacias modeladas. Esse critério, segundo os pesquisadores, limita a comparação com estudos que priorizam uma modelagem realista de espaços ocupados por telhados verdes em áreas urbanas.

A dificuldade de comparação com estudos prévios também pode ser observada no estudo exposto por Paithankar e Taji (2020), que ao utilizar o SWMM para modelar telhados verdes em um complexo de sub-bacias em área urbana da cidade de Kopargaon (Índia), pontuam que o desempenho da LID modelada apresenta sensibilidade à área de cobertura projetada, à inclinação do telhado, ao tipo de cobertura vegetal utilizada, além de apresentar influência do período seco anterior ao cenário modelado. A depender de como essas 
peculiaridades são modeladas, dificulta-se a comparação entre o estudo realizado e estudos anteriores para aferir o grau de sucesso nas abordagens e soluções apresentadas.

Contudo, apesar das limitações, nesse estudo da Bacia PD14, a redução de 4,7\% no escoamento superficial, considerando o cenário com $10 \%$ de área coberta por telhados verdes e tempo de retorno de 5 anos, é coerente com o resultado do estudo apresentado por Mentens et al. (2006).

Na investigação apresentada por Mentens et al. (2006), a aplicação de $10 \%$ de telhados verdes para a área de Bruxelas resultou em uma diminuição no volume de escoamento de 2,7 $\%$ na região, para uma chuva com tempo de retorno de 5 (cinco) anos. O resultado apresentado é próximo ao apresentado na Bacia PD14, considerando condições similares de configuração do modelo.

Ainda sob a perspectiva da similaridade de resultados, mas com o foco no entendimento das condições gerais que atenuam o escoamento superficial, observa-se o estudo realizado Shin e Kim (2015) na bacia urbana de Bangbae-dong (Seul). Nesse estudo, ao se utilizar o PCSWMM para simular a área de cobertura de telhados verdes variando de $50 \%$ a $100 \%$ da área possível na bacia analisada, percebeu-se um aumento considerável na redução do escoamento superficial, variando de $14,7 \%$ a $25,6 \%$.

Tal particularidade também foi observada no estudo da bacia PD14, quando se incrementa a porcentagem de cobertura de telhados verdes de $10 \%$ para $75 \%$. Segundo resultados apresentados, a redução do escoamento superficial varia de 4,7 \% para 38,86 \%, com comportamento coerente com os estudos que observaram a redução dos picos de vazão a partir do aumento da área de implantação de telhados verdes.

Nesse ponto da discussão, é possível apontar resultados semelhantes na investigação realizada por Hamouz et al. (2020), em que se utilizou o PCSWMM para simular bacias urbanas na cidade de Trondheim (Noruega) e verificou-se que os telhados verdes conseguiram reduzir a vazão máxima em 30\% quando a área da cobertura foi duplicada artificialmente em cada uma das sub-bacias modeladas, em simulação a um cenário de adensamento urbano.

A mitigação do escoamento superficial pelo incremento de telhados verdes tem sido assim apontada por diversos estudos, como na área modelada por Yao et al. (2020), que utilizou o SWMM para simular o comportamento hidrológico do escoamento superficial de 12 sub- 
bacias da área urbana de Beijing (China) sob a implementação de telhados verdes na região, encontrando redução significativas do escoamento.

Segundo Yao et al. (2020) os efeitos de redução do escoamento relacionados à implementação de telhados verdes foram claramente diferentes entre as 12 bacias hidrográficas. Esse efeito discrepante se deu por conta das diferentes proporções de telhados verdes em cada sub-bacia modelada. Como conclusão do estudo, observou-se que a maior redução da precipitação pluvial ocorreu em bacias com maior proporção de telhados verdes.

\section{Potencialidades e Limitações}

Embora não tenha sido objeto do modelo aqui apresentado na bacia PD14, espera-se que o desempenho das LIDs (telhados verdes) seja ainda mais eficaz para eventos chuvosos com tempo de concentração menores do que 5 (cinco) anos. Essa assertiva se baseia nos estudos de Ercolani et al. (2018), Mentens et al. (2006); Qin et al. (2013) e Palla e Gnecco (2015) que indicam que os dispositivos são mais eficazes durante os eventos de chuva mais curtos.

No estudo de Ercolani et al. (2018), por exemplo, o impacto dos telhados verdes apresenta maiores taxas de redução do volume escoado considerando o evento modelado com período de 2 (dois) anos. Nessa mesma investigação, os pesquisadores indicaram que não existe redução factível do escoamento superficial, quando o tempo de retorno para o evento chuvoso modelado é de 10 anos.

Essa perspectiva também foi verificada no estudo apresentado por Liu et al. (2020), onde os pesquisadores, ao se utilizar do SWMM para simular $100 \%$ de cobertura de telhados verdes em uma microbacia na cidade de Nanchang (China), observaram que ocorre uma redução significativa da eficiência do telhado verde conforme aumenta-se o período de retorno do evento chuvoso.

Tal particularidade também foi observada por Versini et al. (2015), onde os pesquisadores, ao se utilizar do SWMM para simular uma variabilidade de coberturas de telhados verdes em uma bacia urbana localizada na cidade francesa de Châtillon, indicaram que o uso da LID parece útil para mitigar os efeitos de um evento chuvoso com período de retorno inferior a 10 anos. Para os eventos mais severos, o impacto do telhado verde é marginal e não pode ser usado para resolver questões relacionadas ao escoamento superficial. 
Por conta dessa peculiaridade, deve-se ponderar sobre a integração do telhado verde com outros dispositivos de desenvolvimento de baixo impacto (MENTENS et al., 2006; QIN et al., 2013; PALLA e GNECCO, 2015). Mentens (2006) afirma que a simples implementação de telhados verdes dificilmente resolverá completamente os problemas do escoamento urbano e precisa ser combinado com outras soluções não convencionais de drenagem urbana para potencializar a redução do escoamento superficial.

\section{CONCLUSÕES}

O adiamento do início do escoamento em 16 minutos, a redução do pico de escoamento superficial em $38,86 \%$ e a redução de $12,13 \%$ no volume de água escoada indicam que o cenário com $75 \%$ de telhados verdes instalados pode auxiliar na mitigação dos pontos de falha (alagamento) do sistema de drenagem urbana da bacia. Com ressalva apenas para um levantamento minucioso das condições da bacia em área já urbanizada.

O cenário com $10 \%$ de telhados verdes entrega $30 \%$ de redução do pico de escoamento, atraso de 15 minutos para início do escoamento superficial e redução do volume de água escoada de 4,7 \%. Nesse caso, embora a redução no volume escoado seja menor, a solução na área da bacia é mais exequível.

Ao se observar os hidrogramas produzidos na modelagem nos dois casos propostos, existe um atraso perceptível no início do escoamento superficial e chegada ao ponto de pico. Essa peculiaridade acrescenta resiliência à bacia PD14 frente aos impactos ocasionados pelos eventos de precipitação. Ademais, assim como a redução de volumes apresentada, podem auxiliar na demonstração da utilidade prática do uso de telhados verdes como medida eficaz de controle e mitigação do escoamento superficial em bacias urbanas.

\section{REFERENCIAS}

CIRIA. The SuDS Manual. Escrito por Woods-Ballard, B. et al. London, UK: CIRIA. 2015.

ERCOLANI, G.; CHIARADIA, E. A.; GANDOLFI, C.; CASTELLI, F.; MASSERONI, D. Evaluating performances of green roofs for stormwater runoff mitigation in a high flood risk urban catchment, J. Hydrol. 2018. doi:10.1016/j.jhydrol.2018.09.050.

HAMOUZ, V.; MØLLER-PEDERSEN, P.; MUTHANNA, T. M. Modelling runoff reduction through implementation of green and grey roofs in urban catchments using PCSWMM, Urban Water Journal, 17:9, 813-826, (2020), DOI: 10.1080/1573062X.2020.1828500 
KOURTIS,I.; TSIHRINTZIS,V.; BALTAS, E. Simulation of Low Impact Development (LID) Practices and Comparison with Conventional Drainage Solutions. Proceedings, 2018.

LIU, L.; SUN, L.; NIU, J.; RILEY, W.J. Modeling Green Roof Potential to Mitigate Urban Flooding in a Chinese City. Water 2020, 12, 2082. https://doi.org/10.3390/w12082082.

MAO, X.; JIA, H.; YU, S. L. Assessing the ecological benefits of aggregate LID-BMPs through modelling. Ecol. Model. 2016.

MARTINS, L. G. B. Avaliação do potencial de aplicação de técnicas compensatórias em áreas urbanas consolidadas. 2017. Tese (Doutorado em Hidráulica e Saneamento) - Escola de Engenharia de São Carlos, Universidade de São Paulo, São Carlos, 2017. doi:10.11606/T.18.2018.tde-16042018-103719. Acesso em: 2020-02-03.

MENTENS, J., RAES, D., HERMY, M., Green roofs as a tool for solving the Rainwater runoff problem in the urbanized 21st century. Landsc. Urban Plan. 77 (3), 217-226. 2006. https://doi.org/10.1016/j.landurbplan.2005.02.010.

MIGUEZ, M. G; VERÓL, A. P.; REZENDE, O. M. Drenagem urbana: do projeto tradicional à sustentabilidade. 1. Ed. - Rio de Janeiro: Elsevier, 2016.

MIGUEZ, M. G.; Gregório, L. T.; VERÓL, A. P. Gestão de riscos e desastres hidrológicos. 1.ed. Rio de Janeiro: Elsevier,2018.

MORA-MELIÀ, D.; LÓPEZ-ABURTO, C. S.; BALLESTEROS-PÉREZ, P.; MUÑOZVELASCO, P. Viability of Green Roofs as a Flood Mitigation Element in the Central Region of Chile. Sustainability 2018, 10, 1130. https://doi.org/10.3390/su10041130

PALLA, A., GNECCO, I. Hydrologic modeling of low impact development systems at the urban catchment scale. J. Hydrol. 528, 361-368. 2015. https://doi.org/10.1016/j. jhydrol.2015.06.050.

PAITHANKAR, D. N., TAJI, S. G. Investigating the hydrological performance of green roofs using storm water management model, Materials Today: Proceedings, Volume 32, Part 4, 2020, Pages 943-950, ISSN 2214- 7853, https://doi.org/10.1016/j.matpr.2020.05.085.

PALLA, A.; GNECCO, I.; LA BARBERA, P. Assessing the Hydrologic Performance of a Green Roof Retrofitting Scenario for a Small Urban Catchment. Water 2018, 10, 1052. https://doi.org/10.3390/w10081052

QIN, H.-P., Li, Z.-X., FU, G. The effects of low impact development on urban flooding under different rainfall characteristics. J. Environ. Manage. 129, 577-585. 2013. https://doi.org/10.1016/j.jenvman.2013.08.026. 
SHIN, E.; KIM, H. Analysing Green Roof Effects in an Urban Environment: A Case of Bangbae-dong, Seoul. J. Asian Archit. Build. Eng. 2015, 14, 315-322.

TERESINA. Secretaria Municipal de Planejamento e Coordenação Geral. Plano Diretor de Drenagem Urbana de Teresina. Relatório Final, 2010.

VERSINI, P., D. RAMIER, D., E. BERTHIER, E., GOUVELLO, B. Assessment of the hydrological impacts of green roof: From building scale to basin scale, Journal of Hydrology, Volume 524, 2015, Pages 562-575, ISSN 0022-1694, https://doi.org/10.1016/j.jhydrol.2015.03.020.

YAO, L., WU, Z., WANG, Y., SUN, S., WEI, W., XU, Y., 2020. Does the spatial location of green roofs affect runoff mitigation in small urbanized catchments? J. Environ. Manage. 268, 110707. https://doi.org/10.1016/j.jenvman.2020.110707 